\title{
Le sentiment d'incompétence pédagogique des enseignants en début de carrière et le soutien à l'insertion professionnelle
}

\author{
Stéphane Martineau \\ Annie Presseau \\ Université du Québec àTrois-Rivières
}

\begin{abstract}
Résumé
Ce texte présente le résultats de deux recherches exploratoires menées auprès de treize enseignants débutant dans la carrière et de six directeurs d'établissements scolaires $d u$ niveau secondaire. Nous y démontrons que le sentiment d'incompétence pédagogique vécu par les nouveaux enseignants s'explique à la fois par les logiques dans lesquelles s'inscrivent leurs actions et par un certain nombre de phénomènes de systèmes propre au monde scolaire.
\end{abstract}

\section{Introduction}

Les premières années dans une carrière sont cruciales pour quiconque. Cela est d'autant plus vrai pour la profession enseignante. On qualifie souvent l'entrée dans la carrière de «période de survie ». Le « choc de la réalité » s'accompagne généralement de la sensation d'être vulnérable. Durant leurs premières années, de nombreux enseignants éprouvent de multiples difficultés dans leur quête d'accomplissement professionnel. Ce court texte rend compte des résultats de deux recherches exploratoires menées entre 1999 et 2001. Dans la première, l'objectif général était d'exposer la nature et les caractéristiques du sentiment d'incompétence ressenti par les enseignants nouvellement en exercice dans les écoles secondaires québécoises. Dans la deuxième, nous avons recueilli le point de vue de quelques directeurs d'école.

Stéphane Martineau, Ph.D.- Professor, Départment des sciences de l'éducation, Université du Québec à Trois-Rivières (UQTR), Centre de recherche interuniversitaire sur la formation et la profession enseignante (CRIFPE). Annie Presseau, Ph.D. -

Professeure, Départment des sciences de l'éducation, Université du Québec à Trois-

Rivières (UQTR), Centre de recherche interuniversitaire sur la formation et la profession ensignnante CRIFPE). 
Le sentiment d'incompétence pédagogique

\section{Cadre de référence}

\section{Sur l'insertion professionnelle}

L'enseignant qui débute dans la carrière se trouve devant un double défi. D'abord, il doit réaliser son insertion sur le plan institutionnel. Ensuite, il doit ajuster ou développer rapidement plusieurs savoirs (Tardif \& Lessard, 1999) et plusieurs compétences à la situation concrète d'intervention où il se trouve. Ainsi, tout en apprenant à connaître son lieu de travail et ses collègues, il continue à apprendre son métier. Ce double défi peut facilement le conduire à adopter un comportement traditionnel et peu novateur, en d'autres termes l'amener à se soumettre à la pression de conformité venant du milieu de travail (ce qui, il faut l'avouer, n'est pas obligatoirement une mauvaise chose, la capacité et la volonté d'innovation venant plus tard).

Les recherches portant sur la socialisation professionnelle des enseignants démontrent que l'entrée dans la carrière est une étape déterminante (Zeichner \& Gore, 1990). Le débutant doit se construire une représentation adéquate et fonctionnelle de son environnement de travail et ce, non plus à partir du point de vue d'un élève (qu'il a été) mais à partir de celui d'un enseignant (qu'il est maintenant). En ce sens, l'insertion professionnelle est un véritable processus de transformation identitaire (Martineau \& Corriveau, 2000). Dans ce processus, il n'est pas rare que le nouvel enseignant se sente bousculé dans ses croyances construites depuis son entrée à l'école jusqu'à sa sortie de l'université (Wideen, Mayer-Smith, \& Moon, 1998). Persuadé de pouvoir s'adapter rapidement à son nouvel emploi, il « déchante » souvent très rapidement.

Les premières années dans la profession exercent ainsi une influence considérable sur la suite de la carrière. Dans les cas les plus dramatiques, des débuts difficiles peuvent conduire le nouvel enseignant à abandonner complètement l'enseignement. À l'inverse, oeuvrer dans un contexte de travail agréable et stimulant accroît très souvent le désir d'engagement professionnel. «Les débuts dans l'enseignement jettent les bases de la dynamique motivationnelle qui animera l'enseignant» (Raymond, 2001, p. 23). Par ailleurs, les premières expériences de travail en milieu scolaire fournissent une large part des matériaux qui constituent la base de connaissances pédagogiques personnelles. En ce sens, la période d'insertion au travail n'est pas sans exercer une influence sur le développement professionnel ultérieur du praticien (Huberman, 1989). Ajoutons à cela que cette période de la carrière se fait la plupart du temps, en Amérique du Nord à tout le moins, sous le sceau d'une 
précarité d'emploi qui peut parfois se prolonger pendant plusieurs années (Mukamurera, 1999). Cette situation n'est pas sans entraîner plusieurs désagréments: multiples préparations de cours différents; prise en charge de classes dans divers niveaux; contrat signé à la dernière minute laissant peu de temps pour se préparer; multiples changements de lieu de travail, etc. Dans ces conditions la constitution de la base de connaissances pédagogiques personnelles devient plus ardue. Par exemple, chaque nouveau contrat peut être l'occasion d'un recommencement « à neuf » où le débutant vit plus profondément que l'enseignant régulier la remise en cause de ses connaissances et de ses compétences. Pas étonnant que, dans ces conditions, de trop nombreux enseignants à l'aube de leur carrière se sentent littéralement dépassés par la tâche à accomplir.

\section{Sur le sentiment d'incompétence pédagogique}

Le débat actuel sur la professionnalisation de l'enseignement pose, entre autres, que l'enseignant possède une certaine autonomie professionnelle (malgré l'imposition des programmes scolaires) et dispose de compétences pédagogiques (Gauthier, Desbiens, Malo, Martineau, \& Simard, 1997). Cette autonomie et ces compétences doivent dans la pratique être mobilisées afin de produire un effet sur les apprentissages et la socialisation des élèves: l'effet enseignant (Felouzis, 1997). Par conséquent, il semble légitime de poser la question de la responsabilité de l'enseignant en ce qui concerne l'apprentissage et la socialisation des élèves. Si l'école, en tant qu'institution, ne peut pas éviter de poser cette question, il va sans dire que, tôt ou tard, cette interrogation, les enseignants se la posent eux-mêmes.

L'enseignement est un métier qui exige une grande flexibilité, une pratique exercée dans l'incertitude, où cohabitent diverses dimensions des rapports humains (Perrenoud, 1996). Il ne peut en effet se résumer à une tâche purement cognitive car les variables affectives, relationnelles, stratégiques, éthiques, et autres, le traversent (Tardif, 1993). Or, l'enseignement, ces dernières années, s'est considérablement complexifié notamment en raison de l'alourdissement des tâches des enseignants et de la diversification des clientèles scolaires (Robert \& Tondreau, 1997). Cette situation place l'enseignant devant des circonstances inédites auxquelles sa formation universitaire ne l'avait souvent pas préparé. Et, comme l'a démontré Schütz (1987), notre capacité à faire face aux événements est fortement liée au «stock de connaissances » que nous possédons, lequel s'appuie sur nos expériences antérieures. Dans ce contexte, l'interrogation sur l'effet enseignant acquiert une nouvelle pertinence et la question de la responsabilité du praticien quant aux 
apprentissages et à la socialisation des élèves se pose d'une manière inédite. Cette conjoncture est propice à l'émergence chez les enseignants du sentiment d'être débordé, de n'être pas en mesure de suffire à la tâche, de ne plus pouvoir produire l'effet escompté, bref, de ressentir un sentiment d'incompétence pédagogique.

On a traditionnellement traité l'incompétence professionnelle sous l'angle de la gestion scolaire (Bridges, 1993; Seyfarth, 1996). Selon cette vision, ce sont les fautes professionnelles graves - conduisant potentiellement au renvoi - telles l'incapacité de maintenir le contrôle de la classe ou de traiter les élèves de manière appropriée, l'incapacité à transmettre efficacement la matière, le refus de suivre le programme scolaire, l'abus envers les élèves ou le manque excessif de ponctualité, qui sont analysées. Ces recherches tentent ainsi de dégager un portrait des cas d'incompétence professionnelle afin de déterminer les meilleures mesures à prendre envers les enseignants impliqués. Or, outre le fait que ces recherches n'abordent pas la question du sentiment vécu par ces enseignants, les problèmes soulevés, selon Bridges (1993), ne concerneraient que 5\% du corps professoral. C'est dire que les autres (les 95\%) peuvent être jugés compétents. Cela n'implique toutefois pas que, dans la trame des micro-événements de la salle de classe et de l'école, ceux-ci se sentent toujours à la hauteur de ce qu'on attend d'eux et surtout, de ce qu'ils attendent d'eux-mêmes.

On sait que le sentiment de compétence apparaît comme un élément capital dans l'efficacité de l'enseignant. La psychologie cognitive a en effet montré que la perception de la contrôlabilité de la tâche est une dimension essentielle de la motivation (Tardif, 1992) et cette dernière s'avère une variable incontournable dans la réalisation adéquate d'un travail. On peut donc postuler a contrario que le sentiment d'incompétence est associé au sentiment de ne pas contrôler la tâche à accomplir et qu'il nuira à la motivation au travail. Les premières années du métier étant celles de l'insertion professionnelle et du nécessaire ajustement qu'elles impliquent, les enseignants en début de carrière seront de toute évidence les plus susceptibles à ressentir un tel sentiment d'incompétence. Par exemple, Grossman et Gudmundsdottir (1987) ont montré que l'acquisition de l'expérience en enseignement permet de raffiner et de rendre plus explicites les modèles de l'enseignement d'une matière. Ainsi, les enseignants expérimentés sont plus à même de résoudre les problèmes en classe notamment en raison de leur plus grande capacité à cerner les préconceptions et les stratégies des élèves.

Mais, comment circonscrire le concept d'incompétence pédagogique? Si la définition du concept de compétence pose problème en enseignement (Rey, 1996), il en va bien sûr de même pour son inverse: l'incompétence. 
Toutefois, afin de baliser le terrain il est possible ici de se donner quelques repères. D'abord, on définira l'incompétence pédagogique non pas comme une incapacité physique ou mentale, une faute criminelle (abus, alcoolisme, etc.) ou une faute déontologique au sens de la Loi de l'Instruction publique du Québec (L.R.Q. chapitre 1-13.3) mais en fonction d'une absence ou d'une faiblesse dans le contrôle de la tâche à accomplir (Martineau, Gauthier, \& Desbiens, 2000). Ensuite, cette absence ou cette faiblesse à contrôler une tâche pédagogique sera déterminée non pas a priori en fonction d'une représentation normative de la pratique enseignante mais à partir de l'interprétation que les enseignants font eux-mêmes. L'incompétence pédagogique est ici définie comme étant une absence ou une faiblesse dans le contrôle ou l'exécution de la tâche professionnelle, telle que perçue par le nouvel enseignant lui-même. Par le fait même, le concept de sentiment d'incompétence pédagogique renvoie donc au vécu cognitif et émotionnel lié à l'évaluation de la contrôlabilité de la tâche tel que mis en discours par les enseignants interviewés.

\section{Méthodologie}

Dans les deux recherches exploratoires présentées ici, nous avons eu recours à une approche qualitative reposant sur des entrevues de type semi-directif (Jones, 2000; Selltiz, Wrightsman, \& Cook, 1977). Ces entrevues abordaient les thèmes suivants. Pour les nouveaux enseignants: évaluation de la formation universitaire reçue, expériences d'insertion professionnelle, difficultés rencontrées en enseignement, stratégies pour les surmonter. Pour les directeurs d'écoles: processus d'accueil des nouveaux, problèmes les plus fréquemment rencontrés par les débutants, mesures de soutien offertes, attentes de compétences.

Dans un premier temps, à l'automne 1999, treize enseignants (5 hommes et 8 femmes) occupant un poste à temps plein dans une école secondaire de la grande région de Trois-Rivières, ont été interviewés.

Les répondants, dont l'âge varie entre 24 et 43 ans, avaient cinq années ou moins d'expérience et enseignaient diverses matières (langues, sciences pysiques et humaines, informatique et éducation physique) à tous les degrés du secondaire. Les rencontres ont duré environ $1 \mathrm{~h} 10$.

Dans un deuxième temps, en janvier et février 2001, nous avons interviewé six membres de directions d'écoles. Nos sujets se répartissaient à part égale entre les deux sexes ( 3 hommes, 3 femmes). Les rencontres, plus brèves qu'avec les enseignants novices, ont duré en moyenne 30 minutes. Pour l'ensemble des données, le processus d'analyse de contenu des entrevues s'est 
fait de la manière suivante. Nous avons d'abord procédé à une transcription intégrale des 19 entrevues. Cette procédure fut suivie d'une lecture flottante permettant de repérer les éléments les plus saillants. À une seconde lecture fut réalisée une thématisation plus systématique. Enfin les deux chercheurs ont procédé à la validation de l'analyse sur la base d'un accord inter-juge avoisinant les $90 \%$ pour $20 \%$ du corpus total des entrevues. Il n'a toutefois pas été possible de procéder à une validation écologique des analyses auprès des sujets rencontrés.

\section{Résultats}

Cette section fait état des résultats obtenus. Dans un premier temps nous présentons ceux concernant les enseignants. Puis, dans un deuxième temps, suivent les résultats des entrevues réalisées auprès des directions d'école.

\section{Les enseignants}

Premièrement, le sentiment d'incompétence pédagogique peut se ressentir tout autant dans les tâches liées au travail en classe avec les élèves que dans celles s'effectuant en dehors de cette enceinte protégée (sortie avec son groupe, travail avec les collègues, etc.). Mais, les dimensions relevant de la pratique en classe en présence du groupe d'élèves sont nettement dominantes confirmant en cela se que soutiennent Tardif et Lessard (1999) quant à la centralité du rapport face à face avec les élèves dans la définition du travail par les enseignants. Deuxièmement, lorsqu'il survient en classe, le sentiment d'incompétence peut être associé à la gestion de la matière (dimensions didactiques) ou à la gestion du groupe d'élèves (selon un découpage de la tâche enseignante devenu classique). On note cependant - et cela va dans le sens des recherches sur l'insertion professionnelle - une légère prépondérance des questions liées à la gestion de la classe sauf dans le cas où le sentiment d'incompétence pédagogique prend son origine dans le fait d'enseigner une matière qu' on ne maîtrise pas (par exemple, être formé en mathématiques et biologie et enseigner l'écologie). Troisièmement, et de la même manière, ce sont tout autant des activités pré-actives (préparation des cours), interactives (prestations en classe) ou post-actives (évaluation de son action ou correction des travaux par exemple) (Gauthier, Desbiens, \& Martineau, 1999) qui peuvent être la source de ce type de sentiment: difficultés à construire des outils pédagogiques ou des activités d'apprentissage, sentiment ou impression que l'on « ennuie » le groupe, dilemmes portant sur les modalités d'évaluation, etc. Quatrièmement, 
l'incompétence pédagogique, on l'attribue parfois à soi - manque d'expérience par exemple - ou à une cause extérieure - une formation initiale qui ne prépare pas adéquatement, l'impossibilité de « gérer » la situation adéquatement compte tenu des ressources (matérielles ou humaines) disponibles, ou des élèves trop « problématiques ». Cinquièmement, le sentiment d'incompétence pédagogique ne donne pas toujours lieu à des stratégies pour le surmonter. L'attitude semble souvent être teintée d'un certain fatalisme: «ça va passer avec le temps »; « je ne peux rien y faire ». Toutefois, lorsque les enseignants enclenchent des actions concrètes pour résoudre la difficulté, leurs préférences sont, dans l'ordre: a) consulter un collègue plus expérimenté, b) mettre « les bouchées doubles » pour acquérir des connaissances sur une matière avec laquelle on est moins familier, c) entrer dans un processus d'auto-réflexion sur son action, d) consulter la direction de l'établissement. Il faut cependant constater que la recherche en sciences de l'éducation (à tout le moins ses résultats publiés sous forme d'ouvrages ou d'articles) n'est ressortie à aucun moment comme source de référence pour développer des stratégies de résolution de problèmes liés au travail. De la même manière les enseignants débutants n'ont pas recours à l'expertise des professeurs d'université qui les ont formés. Sixièmement, en tant que représentation et évaluation de soi (comme pédagogue) et de l'efficacité de son action face à une situation donnée, le sentiment d'incompétence pédagogique apparaît étroitement lié à une représentation normative du bon enseignant construite par les nouveaux enseignants. Les termes les plus souvent employés pour qualifier cette représentation sont: guide, éducateur, modèle, initiateur, aide. On constatera qu'il y a ici reprise d'une partie de la terminologie en vogue dans le cadre des réformes en cours dans plusieurs systèmes d'éducation dont celui du Québec (Martineau \& Gauthier, 2002). En ce sens, le sentiment d'incompétence pédagogique est également associé à la pression à la conformité qui vient du milieu de travail.

Dans une certaine mesure, le contenu des entrevues auprès des nouveaux enseignants nous porte à croire que la socialisation au travail apparaît comme étant tout à la fois une « initiation » (au sens anthropologique du terme) à la culture professionnelle et une « conversion » (au sens religieux) du sujet à une nouvelle représentation du monde et de soi. Les sujets interviewés ont vécu durant leurs premières années un processus complexe de transformation en profondeur de leur identité professionnelle. D'étudiants en formation des maîtres, ils sont «presque » devenus des enseignants au plein sens du terme. La complète reconnaissance par les pairs plus expérimentés ne semble pas en effet tout à fait acquise. Même si l'abandon de la probation depuis 1998 a contribué à clarifier le statut des débutants (Lévesque \& Gervais, 2000), les nouveaux enseignants ne se sentent pas totalement perçus comme des « enseignants 
accomplis ». Ils expriment donc à de multiple reprises leur besoin de reconnaissance de la part des enseignants chevronnés. Ils veulent être perçus comme des collègues à part entière. Ils répugnent ainsi à être traités de manière condescendante mais reconnaissent qu'ils sont encore en apprentissage. Pour revenir au sentiment d'incompétence pédagogique, disons que celui-ci ne semble pas avoir été très profond. Ainsi, les problèmes évoqués ne sont pas insurmontables et leur gravité n'ébranle pas la confiance en soi des enseignants. Par exemple, aucun sujet n'a remis en cause sa carrière. Leurs représentations, leurs attitudes, leurs croyances, leurs valeurs n'ont pas été modifiées de manière drastique par les événements « dérangeants » vécus en classe. D'une certaine manière on pourrait presque dire que le sentiment d'incompétence pédagogique relève en quelque sorte du processus «normal » d'apprentissage du métier.

À travers l'expression de leur sentiment d'incompétence pédagogique, les enseignants rencontrés expriment aussi leurs besoins en matière de soutien à l'insertion professionnelle. Ces besoins concernent avant tout les savoirs pédagogiques. Ainsi, ils souhaitent obtenir des conseils sur les meilleures stratégies pour maintenir la discipline en classe ou encore qu'on leur donne de « bons trucs » pour susciter la motivation à apprendre chez les élèves. De plus, ils souhaiteraient recevoir une formation pour mieux maîtriser l'évaluation formative et sommative des travaux des élèves. En définitive, il semble que le sentiment d'incompétence pédagogique soit alimenté, du moins en partie, par le peu de soutien à l'insertion professionnelle dispensé par les directeurs d'établissements. Dans un sens, l'organisation individualiste de l'enseignement perdure (Tardif \& Lessard, 1999) et l'insertion professionnelle se fait sous le sceau de « la débrouille ». Néanmoins, malgré la faible appréciation de la formation initiale, malgré le peu de soutien de la part des collègues et de la direction, malgré une population étudiante jugée de plus en plus « problématique » au regard des valeurs et des normes scolaires, les enseignants semblent considérer ne pas trop mal s'en tirer. Tout semble se passer comme s'ils intégraient très tôt une vision du métier où il est légitime de ne pas « être toujours à la hauteur ». Cette vision du métier leur permet peut-être de supporter les moments difficiles sans remise en question radicale (voire même sans aucune remise en question).

\section{Les directeurs d'établissement}

Les éléments qui suivent font état des points de convergence du discours des directions quant aux compétences attendues envers les enseignants novices. Nous avons regroupé ces compétences en cinq grandes catégories. 
A. Les compétences en gestion de la classe. Les directions souhaitent que les nouveaux enseignants maîtrisent les compétences par rapport à la gestion de classe afin d'intervenir adéquatement en matière de la discipline.

B. Les compétences en gestion de la matière. On s'attend à ce qu'ils puissent préparer, planifier et transmettre adéquatement la matière qu'ils enseignent. Ainsi, les directions exigent des nouveaux enseignants qu'ils fassent preuve d'une excellente maîtrise des contenus à enseigner. Par ailleurs, on souhaite qu'ils aient, dès leur embauche, des compétences pédagogiques actualisées, c'est-à-dire adaptées aux nouvelles réalités éducatives (réforme, nouveau curriculum, régime pédagogique, etc.). Les directions s'attendent aussi à ce que les nouveaux enseignants possèdent une bonne connaissance de l'usage des technologies informatiques afin de les utiliser dans leurs cours et y entrainer les élèves. Enfin on exige qu'ils soient familiers avec les outils pédagogiques et les techniques d'enseignement intégrées comme l'apprentissage coopératif, la pédagogie par projet, et autres.

C. Les compétences en relations interpersonnelles. Les directions s'attendent à ce que les enseignants nouvellement embauchés soient capables d'établir de bons contacts avec les élèves ainsi qu'avec leurs collègues. On exige aussi qu'ils soient capables de s'impliquer, de partager et de participer à la vie de l'école.

D. Les compétences au travail en équipe. Bien que les directeurs rencontrés n'aient pas été très explicites à cet égard quant aux modalités d'actualisation de cette compétence, ils ont tout de même clairement exprimé leur exigence en terme de capacité du nouvel enseignant à travailler en équipe professorale tout en développant un style personnel. 
E. Les compétences éthiques. Les enseignants débutants devraient être des gens disciplinés, par rapport à leur profession et surtout par rapport au code de vie de l'école. Ils doivent se distinguer des élèves par leurs comportements, c'est-à-dire qu'ils doivent être des modèles pour les élèves (une bonne tenue en classe, habillement correct, bonne diction, respect des règlements de l'école) afin de ne pas être confondus avec eux.

F. Le processus d'accueil des débutants. En ce qui concerne le processus d'accueil des nouveaux enseignants, disons que celui-ci est loin d'être formalisé ou planifié. Les propos recueillis auprès de toutes les directions d'école convergent sur les éléments suivants. Les novices ont droit à une période d'accueil et de rencontres avec la direction de l'école ou par un membre du personnel de la direction (connaissance mutuelle, échange sur le vécu de l'école, sur les activités, la culture organisationnelle de l'école). Ensuite, les nouveaux sont présentés à l'équipe enseignante. S'ajoute régulièrement à cela une visite de l'école durant laquelle on explique entre autres les règlements à respecter, le programme d'études, le fonctionnement de l'établissement, le projet éducatif. Enfin, dans certain cas, il y a parrainage ou jumelage des nouveaux avec des « anciens » selon le domaine et le niveau d'enseignement (durée entre six et douze mois). Le parrain a pour tâche d'initier le nouveau à la préparation des cours, au suivi des élèves, au travail à faire pendant et après l'enseignement. Dans l'ensemble, les directions d'écoles négligent la question de la mise en place de mesures de soutien formel.

G. Les « incompétences pédagogiques ». Quant aux incompétences pédagogiques relevées par les directions chez les nouveaux enseignants, les entrevues laissent entrevoir les éléments qui suivent. D'abord, les débutants éprouvent régulièrement une certaine incapacité à assurer la discipline en classe, c'est-à-dire, qu'ils ont de la difficulté à maîtriser un groupe d'élèves (parce que, aux 
dires des directions, ils se font « trop amis » avec les élèves et parce qu'ils ne sont pas souvent disciplinés euxmêmes). On déplore aussi leurs réticences à demander de l'aide quand ils sont confrontés à des problèmes d'ordre professionnel, réticences qui peuvent toutefois s'expliquer par la crainte d'être jugés incompétents ou incapables. De la même manière, on relève leurs difficultés à remplir adéquatement les tâches de planification et de préparation des cours. De plus, leurs connaissances pédagogiques seraient souvent inadéquates au regard des programmes scolaires. Enfin, les débutants dans la carrière enseignante présenteraient de grandes lacunes quant à la culture générale (celle-ci n'est toutefois jamais clairement définie ni caractérisée par les directions).

\section{Conclusion}

Rappelons d'abord que nous rendons compte ici de deux recherches exploratoires et qu'à cet égard nos conclusions ne peuvent qu'être très prudentes et provisoires. D'abord, on peut dire que les débutants semblent ne pas trop mal se débrouiller durant leurs premiers pas dans la carrière. On constate ainsi que le sentiment d'incompétence ressenti par les treize enseignants rencontrés a été peu profond. Il fut en réalité vécu en relation avec des événements ponctuels et non pas en rapport à des événements récurrents. Par ailleurs, les stratégies de remédiation, lorsqu'elles existent, peuvent être qualifiées d'endogamiques à savoir que l'enseignant recherche des solutions en lui-même ou à l'intérieur de l'école (auprès d'un collègue) et exclut ainsi les sources extérieures telles les écrits scientifiques ou ses anciens professeurs à l'université. Ce comportement confirme le peu d'autorité qu'exercent les chercheurs et les formateurs en sciences de l'éducation auprès des praticiens de l'enseignement. Toutefois, selon nous, ce qui interpelle le plus fortement le monde de l'éducation c'est l'attitude que nous qualifierions de fataliste face aux situations professionnelles où le débutant se sent incompétent. Les nouveaux enseignants semblent en effet avoir développé très tôt une représentation de la profession comme étant un «métier impossible » ce qui pourrait, dans une certaine mesure, inhiber leur désir de trouver des solutions et entretenir ainsi une certaine apathie. Par ailleurs, en ce qui concerne plus globalement le processus d'insertion professionnelle, on peut dire qu'il se vit sur le mode informel plutôt que formel. Les directions semblent en réalité offrir peu de 
soutien à cet effet. De la même manière, les collègues apparaissent globalement peu présents en matière d'aide. En somme, le sentiment d'incompétence pédagogique renvoie à la fois à des phénomènes de systèmes (des conditions d'entrée difficiles, les « mauvais groupes », les pires horaires, la précarité d'emploi, une culture de l'individualisme où c'est « chacun pour soi dans sa classe », l'absence de structures d'insertion précises et formelles) et des logiques d'acteurs ( des attentes élevées mais implicites des directions, le fatalisme lié à une certaine représentation du travail, la recherche de solutions dans un spectre restreint de ressources).

En ce sens, les résultats de ces deux recherches exploratoires confirment les tendances observées dans des recherches récentes tant dans le champ de l'analyse du travail enseignant (Tardif \& Lessard, 1999) que dans celui de l'insertion professionnelle (Hétu, Lavoie, \& Baillauquès, 1999; Mukamurera, 1998).

\section{Références}

Bridges, E.M. (1993). The incompetent teacher. Managerial responses. Washington, D.C.: The Falmer Press.

Felouzis, G. (1997). L'efficacité des enseignants. Paris: PUF.

Gauthier, C., Desbiens, J.-F., Malo, A., Martineau, S., \& Simard, D. (1997). Pour une théorie de la pédagogie. Recherches contemporaines sur le savoir des enseignants. Sainte-Foy: Les Presses de l'Université Laval.

Gauthier, C., Desbiens, J.-F., \& Martineau, S. (1999). Mots de passe pour mieux enseigner. Sainte-Foy: Les Presses de l'Université Laval.

Grossman, P.L., \& Gudmundsdottir, S. (1987). Teachers and texts: An expert/ novice study in English (Knowledge Growth in a Profession Publication Series). Stanford, CA: Stanford University, School of Education.

Hétu, J.-C., Lavoie, M., \& Baillauquès, S. (eds.) (1999). Jeunes enseignants et insertion professionnelle. Un processus de socialisation? de professionnalisation? de transformation?. Bruxelles: De Boeck.

Huberman, A.M. (1989). La vie des enseignants. Évolution et bilan d'une profession. Neuchâtel/Paris: Delachaux \& Niestlé.

Jones, R.A. (2000). Méthodes de recherche en sciences humaines. Bruxelles: De Boeck.

Lévesque, M., \& Gervais, C. (2000). L'insertion professionnelle: une étape à réussir dans le processus de professionnalisation de l'enseignement. Éducation Canada, 40(1), 12-15. 
Martineau, S., \& Corriveau, G. (2000). Vers une meilleure compréhension du sentiment d'incompétence pédagogique chez les enseignants en insertion professionnelle au secondaire. Formation et Profession, 6(3), 5-8.

Martineau, S., \& Gauthier, C. (2002). Évolution des programmes scolaires au Québec: un aperçu historique pour mieux comprendre la réforme actuelle. In C. Gauthier \& D. Saint-Jacques (Eds.), La réforme des programmes scolaires au Québec (pp. 1-21). Québec: Les Presses de l'Université Laval.

Martineau, S., Gauthier, C., \& Desbiens, J.-F. (2000). Ce n'est pas toujours la faute à El Niño. À propos de l'incompétence en enseignement. In C. Lessard \& C. Gervais (Eds.), L'évaluation des nouveaux programmes de formation des maîtres: une compétence à développer (pp. 299-332). Montréal: Les Publications de la Faculté des sciences de l'éducation.

Mukamurera, J. (1998). Étude du processus d'insertion professionnelle de jeunes enseignants à partir du concept de trajectoire. Thèse de doctorat. Sainte-Foy: Université Laval.

Mukamurera, J. (1999, avril-mai). Les trajectoires d'insertion de jeunes profs au Québec. Vie Pédagogique, 111, 24-27.

Perrenoud, P. (1996). Enseigner: agir dans l'urgence, décider dans l'incertitude. Paris: ESF.

Raymond, D. (2001). Processus et programmes d'insertion professionnelle des enseignants du collégial. Pédagogie collégiale, 14(3), 22-27.

Rey, B. (1997). Les compétences transversales en question. Paris: ESF.

Robert, M. \& Tondreau, J. (1997). L'école québécoise. Débats, enjeux et pratiques sociales. Une analyse sociale de l'éducation pour la formation des maîtres. Montréal: CEC.

Schütz, A. (1987). Le chercheur et le quotidien. Paris: Méridiens Klincksieck.

Selltiz, C., Wrightsman, I.S., \& Cook, S.W. (1977). Les méthodes de recherche en sciences sociales. Montréal: HRW.

Seyfarth, J.T. (1996). Personnel management for effective schools ( $2^{\mathrm{e}}$ éd.). Boston: Allyn and Bacon.

Tardif, J. (1992). Pour un enseignement stratégique. L'apport de la psychologie cognitive. Montréal: Logiques.

Tardif, M. (1993). Savoirs et expérience chez les enseignants de métier. In H. Hensler (Ed.), La recherche en formation des maîtres. Détour ou passage obligé sur la voie de la professionnalisation? (p. 53-86). Sherbrooke: CRP.

Tardif, M., \& Lessard, C. (1999). Le travail enseignant au quotidien. Contribution à l'étude du travail dans les métiers et les professions 
d'interactions humaines. Sainte-Foy: Les presses de l'Université Laval.

Wideen, M., Mayer-Smith, J., \& Moon, B. (1998). A critical analysis of research on learning to teach: Making the case for an ecological perspective on inquiry. Review of educational research, 68(2), 130178.

Zeichner, K.M. \& Gore, J.M. (1990). Teacher socialization. In W.R. Houston, M. Huberman, \& J.K. Sikula (eds.), Handbook of research on teacher education (pp. 329-348). New York: MacMillan. 